\title{
On the Robustness of Global Optima and Stationary Solutions to Stochastic Mathematical Programs with Equilibrium Constraints, part 2: Applications
}

\author{
C. Cromvik* M. Patriksson ${ }^{\dagger}$ \\ Communicated by F. Giannessi
}

${ }^{*}$ Mathematical Sciences, Chalmers University of Technology and Mathematical Sciences, University of Gothenburg, SE-41296 Gothenburg, Sweden. E-mail: christoffer.cromvik@chalmers.se

${ }^{\dagger}$ Corresponding author. Mathematical Sciences, Chalmers University of Technology and Mathematical Sciences, University of Gothenburg, SE-412 96 Gothenburg, Sweden. E-mail: mipat@chalmers.se 
Abstract In a companion paper (Cromvik and Patriksson, On the Robustness of Global Optima and Stationary Solutions to Stochastic Mathematical Programs with Equilibrium Constraints, part 1: Theory, Journal of Optimization Theory and Applications, 2010, to appear) the mathematical modeling framework SMPEC was studied; in particular, global optima and stationary solutions to SMPECs were shown to be robust with respect to the underlying probability distribution under certain assumptions. Further, the framework and theory were elaborated to cover extensions of the upper-level objective: minimization of the conditional value-at-risk $(\mathrm{CVaR})$ and treatment of the multiobjective case. In this paper, we consider two applications of these results: a classic traffic network design problem, where travel costs are uncertain, and the optimization of a treatment plan in intensity modulated radiation therapy, where the machine parameters and the position of the organs are uncertain. Owing to the generality of SMPEC, we can model these two very different applications within the same framework. Our findings illustrate the large potential in utilizing the SMPEC formalism for modeling and analysis purposes; in particular, information from scenarios in the lower-level problem may provide very useful additional insights into a particular application.

Keywords: Stochastic mathematical program with equilibrium constraints, Solution stability and robustness, Traffic network design, Intensity modulated radiation therapy, Sample average approximation 


\section{Introduction}

The framework known as stochastic mathematical program with equilibrium constraints (SMPEC) was introduced in [1] as an extension of the MPEC framework of hierarchical optimization models, in order to incorporate the uncertainty of data that one often must face in applications. Since then, it has found applications in many contexts, such as economics (e.g., [2,3]), engineering sciences (e.g., [4,5]), and transportation science (e.g., [6]). The companion paper [7] provides new results on the robustness of solutions to this class of problems. The objective of this paper is to illustrate the potential utilization of these results through two applications. The first is within traffic network design, which is a classic topic (see e.g. [6,8] and references therein). The second, however, appears to be new: we present an SMPEC model for robust treatment planning in intensity modulated radiation therapy (IMRT), where the machine parameters as well as the position of the organs are uncertain. With this model, we are approaching a robust and in fact deliverable treatment plan.

The remainder of the paper is organized as follows. In Section 2, we summarize briefly the main results from [7]. In Section 3, we provide a small-scale application of the robust design of a traffic network, based on the classic network of Braess. In Section 4, we provide a numerical example of a treatment plan, which accounts for both position uncertainty and the uncertainties in the radiation delivery from a treatment machine.

\section{Robustness of Solutions to the SMPEC}

Let $f: \mathbb{R}^{n} \times \mathbb{R}^{m} \rightarrow \mathbb{R}, y \in \mathbb{R}^{m}, C \subseteq \mathbb{R}^{m}$ be a polyhedron, let $F(x, \cdot): C \rightarrow \mathbb{R}^{m}$ be smooth, and let $N_{C}: \mathbb{R}^{m} \rightrightarrows \mathbb{R}^{m}$ be the standard normal cone mapping,

$$
N_{C}(y):= \begin{cases}\left\{z \in \mathbb{R}^{m} \mid z^{\mathrm{T}}(w-y) \leq 0, w \in C\right\}, & \text { if } y \in C, \\ \emptyset, & \text { otherwise. }\end{cases}
$$

Let $(\Omega, \Theta, \mathrm{P})$ be a complete probability space and consider the problem

$$
\begin{aligned}
\left(\mathrm{SMPEC}_{\Omega}\right) \min _{(x, y(\cdot))} & \mathrm{E}_{\omega}[f(x, y(\omega), \omega)]:=\int_{\Omega} f(x, y(\omega), \omega) P(d \omega), \\
\text { s.t. } \quad & x \in X, \\
& -F(x, y, \omega) \in N_{C}(y), \quad P \text {-a.s., }
\end{aligned}
$$

where $y: \Omega \rightarrow \mathbb{R}^{m}$ is a random element of the probability space $(\Omega, \Theta, \mathrm{P})$. We also introduce $S: \mathbb{R}^{n} \times \Omega \rightrightarrows \mathbb{R}^{m}$, which defines the set of solutions to the lower-level parametric variational inequality problem,

$$
S(x, \omega):=\left\{y \in \mathbb{R}^{m} \mid-F(x, y, \omega) \in N_{C}(y)\right\} .
$$

We next summarize briefly the main technical content of [7]. 


\subsection{Stability of Global Solutions and Stationary Points}

\section{Assumption A}

(A1) The mapping $S(x, \cdot)$ is measurable for any $x$.

(A2) The set $X$ is closed and the mapping $x \mapsto S(x, \omega)$ is closed for almost any $\omega \in \Omega$.

(A3) The function $f$ is continuous in $(x, y)$, measurable in $\omega$, uniformly weakly coercive with respect to $x$ over the set $X$, and bounded from below by a $(\Theta, \mathrm{P})$-integrable function.

(A4) The set $S\left(x_{0}, \omega\right)$ is nonempty for some $x_{0} \in X$ and almost any $\omega \in \Omega$.

The existence of optimal solutions under Assumption A is established in [9].

Let $\left\{P_{k}\right\}$ be a sequence of probability measures defined on $\mathcal{B}(\Omega)$, and denote by $\left(\mathrm{SMPEC}_{\Omega}\right)^{k}$ the problem defined by $\left(\mathrm{SMPEC}_{\Omega}\right)$ with the measure $P$ replaced by $P_{k}$.

Theorem 2.1 (Global Stability of Optimal Solutions) Let Assumption (A) hold, suppose that the mapping $F(x, \cdot, \omega)$ is strictly monotone in $y$ for each $x \in X$ and $\omega \in \Omega$, and that the sequence $\left\{P_{k}\right\}$ of probability measures weakly converges to $P$. Also suppose that, for each $k,\left(x^{k}, y^{k}(\cdot)\right)$ solves $\left(\mathrm{SMPEC}_{\Omega}\right)^{k}$. Then, each limit point (there is at least one) of the sequence $\left\{\left(x^{k}, y^{k}(\cdot)\right)\right\}$ is an optimal solution to $\left(\mathrm{SMPEC}_{\Omega}\right)$.

\section{Assumptions B}

(B1) The function $f$ is Lipschitz continuous in $(x, y)$.

(B2) The mapping $F(\cdot, \cdot, \omega)$ is continuously differentiable and $F(x, \cdot, \omega)$ is uniformly strongly monotone on $C$ for each $x \in X$ and $\omega \in \Omega$.

(B3) $X=\left\{x \in \mathbb{R}^{n} \mid g_{i}(x) \leq 0, i=1, \ldots, p\right\}$ and each function $g_{i}$ is continuously differentiable.

(B4) The Mangasarian-Fromovitz constraint qualification (MFCQ) holds for all $x \in X$.

If Assumptions (B1) and (B2) hold, then ( [10]) there exists a locally Lipschitz continuous, single-valued solution map $(x, \omega) \mapsto \sigma(x, \omega)$ with

$$
y=\sigma(x, \omega), \quad \sigma(x, \omega) \in S(x, \omega) .
$$

With this property, we can rewrite $\left(\mathrm{SMPEC}_{\Omega}\right)$ as the one-level problem

$$
\begin{aligned}
\left(\operatorname{SNLP}_{\Omega}\right) \quad \min _{x} & \mathrm{E}_{\omega}[f(x, \sigma(x, \omega), \omega)]:=\int_{\Omega} f(x, \sigma(x, \omega), \omega) P(d \omega), \\
\text { s.t. } \quad & x \in X
\end{aligned}
$$

and correspondingly $\left(\operatorname{SNLP}_{\Omega}\right)^{k}$ is obtained from $\left(\operatorname{SNLP}_{\Omega}\right)$ by replacing $P$ with $P_{k}$.

Theorem 2.2 (Stability of Stationary Solutions) Let Assumptions (A) and (B) hold, suppose that the sequence $\left\{P_{k}\right\}$ of probability measures weakly converges to $P$ and is upper bounded by a measurable function, and that for each $k,\left(x^{k}, y^{k}(\cdot)\right)$ is a Clarke stationary solution to $\left(\mathrm{SNLP}_{\Omega}\right)^{k}$. Then, each limit point (there is at least one) of the sequence $\left\{\left(x^{k}, y^{k}(\cdot)\right)\right\}$ is a weakly Clarke stationary solution to $\left(\operatorname{SNLP}_{\Omega}\right)$. 


\subsection{Convergence of Sample Average Approximation Schemes}

We use the reformulation of $(\mathrm{SMPEC})_{\Omega}$ into $\left(\mathrm{SNLP}_{\Omega}\right)$, and consider the problem

$$
\begin{aligned}
(\mathrm{SNLP})^{N} & \min _{x} \quad \hat{f}_{N}:=\frac{1}{N} \sum_{k=1}^{N} f\left(x, \sigma\left(x, \omega^{k}\right), \omega^{k}\right), \\
\text { s.t. } & x \in X .
\end{aligned}
$$

\section{Assumption C}

(C1) The set $X$ is bounded and convex.

(C2) The function $f(\cdot, \sigma(\cdot, \omega), \omega)$ is regular (i.e., $f$ is directionally differentiable and the directional derivative coincides with the Clarke directional derivative) at $x$ for almost any $\omega \in \Omega$.

Theorem 2.3 (Convergence of Optimal Solutions) Let Assumptions (A), (B1)-(B2), (C1) hold. For each $N$, let $\left(x_{N}, y_{N}(\cdot)\right)$ be an optimal solution to $(\mathrm{SNLP})^{N}$. Then, each limit point (there is at least one) of the sequence $\left\{x_{N}\right\}$ is an optimal solution to $\left(\mathrm{SNLP}_{\Omega}\right)$.

Theorem 2.4 (Convergence of Stationary Solutions) Let Assumptions (A), (B), (C) hold. For each $N$, let $\left(x_{N}, y_{N}(\cdot)\right)$ be a stationary solution to $(\mathrm{SNLP})^{N}$. Then, each limit point (there is at least one) of the sequence $\left\{x_{N}\right\}$ is a stationary solution to $\left(\mathrm{SNLP}_{\Omega}\right)$.

Since $\left(\operatorname{SNLP}_{\Omega}\right)$ is a reformulation of $\left(\mathrm{SMPEC}_{\Omega}\right)$, the above theorems state that we also have convergence to global optima and stationary solutions for the corresponding discretized problem

$$
\begin{aligned}
(\mathrm{SMPEC})^{N} \quad \min _{x} \quad \hat{f}_{N}:=\frac{1}{N} \sum_{k=1}^{N} f\left(x, y^{k}, \omega^{k}\right), \\
\text { s.t. } \quad x \in X, \\
\quad-F\left(x, y^{k}, \omega^{k}\right) \in N_{C}\left(y^{k}\right), \quad k=1, \ldots, N .
\end{aligned}
$$

\subsection{Extensions to a Risk Objective Function and to Multiple Objectives}

We consider next the SMPEC where the expected value in the objective function is replaced by an expression for conditional value-at-risk (CVaR) at level $\beta$

$$
\begin{aligned}
\left.(\mathrm{SRPEC})_{\Omega}\right) \quad \min _{(x, y(\cdot), \gamma)} & \gamma+\frac{1}{1-\beta} \int_{\Omega}[f(x, y(\omega), \omega)-\gamma]_{+} P(d \omega), \\
\text { s.t. } \quad & x \in X, \\
& -F(x, y, \omega) \in N_{C}(y), \quad P \text {-a.s. }
\end{aligned}
$$


and analogously for the problem $\left(\mathrm{SRPEC}_{\Omega}\right)^{k}$. In line with Theorem 2.1, we can establish robustness of global optima; by reformulating SMPEC into one-level problems,

$$
\begin{aligned}
\left(\operatorname{SRNLP}_{\Omega}\right) \quad \min _{(x, \gamma)} & \gamma+\frac{1}{1-\beta} \int_{\Omega}[f(x, \sigma(x, \omega), \omega)-\gamma]_{+} P(d \omega), \\
\text { s.t. } & x \in X,
\end{aligned}
$$

we can also establish robustness of stationary solutions as in Theorem 2.2, and the convergence of the SAA scheme as in Theorems 2.3 and 2.4; see [7] for details.

We next define the multiple objective version of the SMPEC, the SMOPEC, for $q$ objectives as that to

$$
\begin{aligned}
\left(\mathrm{SMOPEC}_{\Omega}\right) \min _{(x, y(\cdot))} & \left(\mathrm{E}_{\omega}\left[f_{1}(x, y(\omega), \omega)\right], \ldots, \mathrm{E}_{\omega}\left[f_{q}(x, y(\omega), \omega)\right]\right), \\
\text { s.t. } \quad x \in X, & \\
& -F(x, y, \omega) \in N_{C}(y), \quad \text { P-a.s. }
\end{aligned}
$$

and correspondingly $\left(\mathrm{SMOPEC}_{\Omega}\right)^{k}$ is obtained by replacing $P$ with $P_{k}$.

Theorem 2.5 (Stability of Weakly Pareto-Optimal Solutions to $\left(\mathrm{SMOPEC}_{\Omega}\right)$ ) Let Assumption (A) hold, suppose that the mapping $F(x, \cdot, \omega)$ is strictly monotone for each $x \in X$ and $\omega \in \Omega$, that $\left(\mathrm{SMOPEC}_{\Omega}\right)$ is a convex problem, and that the sequence $\left\{P_{k}\right\}$ of probability measures weakly converges to $P$. Also, suppose that, for each $k,\left(x^{k}, y_{k}(\cdot)\right)$ is a weakly Pareto-optimal solution to $\left(\mathrm{SMOPEC}_{\Omega}\right)^{k}$. Then, each limit point (there is at least one) of the sequence $\left\{\left(x^{k}, y^{k}(\cdot)\right)\right\}$ is a weakly Pareto-optimal solution to $\left(\mathrm{SMOPEC}_{\Omega}\right)$.

To establish stability without a convexity assumption, we reformulate $\left(\mathrm{SMOPEC}_{\Omega}\right)$ and $\left(\mathrm{SMOPEC}_{\Omega}\right)^{k}$ as one-level problems by treating $y$ as a function of $x$ and $\omega, y=\sigma(x, \omega)$. This is possible if, in addition to the assumptions in Theorem 2.5, Assumptions (B1) and (B2) hold. We denote the reformulations by $\left(\mathrm{SMONLP}_{\Omega}\right)$ and $\left(\mathrm{SMONLP}_{\Omega}\right)^{k}$, respectively, where the first problem has the following appearance:

$$
\begin{aligned}
\left(\operatorname{SMONLP}_{\Omega}\right) \quad \min _{x} & \left(\mathrm{E}_{\omega}\left[f_{1}(x, \sigma(x, \omega), \omega)\right], \ldots, \mathrm{E}_{\omega}\left[f_{q}(x, \sigma(x, \omega), \omega)\right]\right), \\
\text { s.t. } & x \in X
\end{aligned}
$$

Theorem 2.6 (Stability of Weakly Pareto-Stationary Solutions to $\left(\mathrm{SMONLP}_{\Omega}\right)$ ) Let Assumptions (A), (B), (C2) hold, suppose that the sequence $\left\{P_{k}\right\}$ of probability measures weakly converges to $P$, and that, for each $k,\left(x^{k}, y^{k}(\cdot)\right)$ is a weakly Pareto-stationary solution to $\left(\mathrm{SMONLP}_{\Omega}\right)^{k}$. Then, each limit point (there is at least one) of the sequence $\left\{\left(x^{k}, y^{k}(\cdot)\right)\right\}$ is a weakly Pareto-stationary solution to $\left(\operatorname{SMONLP}_{\Omega}\right)$.

\section{First Application: Traffic Network Design}

We consider a road traffic model. The network is represented by a strongly connected graph $\mathcal{G}=(\mathcal{V}, \mathcal{E})$, where $\mathcal{V}$ is the set of nodes and $\mathcal{E}$ is the set of directed links. For each origin-destination (OD) pair $(p, q) \in \mathcal{C}$ with $\mathcal{C} \subset \mathcal{V} \times \mathcal{V}$, there is a transportation demand. 
Each route $r \in \mathcal{R}_{p q}$ joining the OD pair $(p, q)$ has an associated flow $h_{r}$ and a travel cost $c_{r}$.

We assume that the design parameter $x \in \mathbb{R}^{n}$ influences the travel cost and the demand. The travel cost function has the form $c(x, \cdot): \mathbb{R}_{+}^{|\mathcal{R}|} \rightarrow \mathbb{R}^{|\mathcal{R}|}$, where $|\mathcal{R}|$ is the total number of routes. The demand for each OD pair depends on the travel cost and the demand function has the form $d(x, \cdot): \mathbb{R}^{|\mathcal{C}|} \rightarrow \mathbb{R}_{+}^{|\mathcal{C}|}$.

Wardrop's user equilibrium condition [11] states that, for each OD pair, the travel cost for all routes utilized must be equal and minimal. Since the flow is nonnegative, this condition can be formulated as a complementarity condition. Let $\pi_{p q}$ be the minimum travel cost for the OD pair $(p, q)$. The equilibrium condition is that

$$
0 \leq h_{r} \perp c_{r}(x, h)-\pi_{p q} \geq 0, \quad r \in \mathcal{R}_{p q}, \quad(p, q) \in \mathcal{C},
$$

where $a \perp b$ denotes the condition $a^{\mathrm{T}} b=0$. Utilizing a route-OD pair incidence matrix $\Gamma \in \mathbb{R}^{|\mathcal{R}| \times|\mathcal{C}|}$, we can express demand feasibility as follows:

$$
\Gamma^{\mathrm{T}} h=d(x, \pi)
$$

Combining (1) and (2), we characterize the user equilibrium flows as a mixed complementarity problem $(\mathrm{MCP})$,

$$
\begin{aligned}
0^{|\mathcal{R}|} \leq h \perp c(x, h)-\Gamma \pi & \geq 0^{|\mathcal{R}|}, \\
\Gamma^{\mathrm{T}} h & =d(x, \pi) .
\end{aligned}
$$

If we assume that the travel cost is positive, then (3) can instead be formulated as the following nonlinear complementarity problem $(\mathrm{NCP})([12,13])$ :

$$
\begin{gathered}
0^{|\mathcal{R}|} \leq h \perp c(x, h)-\Gamma \pi \geq 0^{|\mathcal{R}|}, \\
0^{|\mathcal{C}|} \leq \pi \perp \Gamma^{\mathrm{T}} h-d(x, \pi) \geq 0^{|\mathcal{C}|} .
\end{gathered}
$$

We can also provide a link flow representation of the user equilibrium condition. Let $v \in \mathbb{R}^{|\mathcal{E}|}$ be a vector of link flows and let $t_{l}(x, v)$ be the link travel cost for $l \in \mathcal{E}$. If we assume that the travel costs are additive and introduce a route-link incidence matrix $\Lambda \in\{0,1\}^{|\mathcal{E}| \times|\mathcal{R}|}$, the link travel cost is related to the route travel cost through the relation $c(x, h)=\Lambda^{\mathrm{T}} t(x, v)$. Also, to have flow conservation, we require that

$$
v=\Lambda h
$$

The main objective in a network design problem is to influence the travel costs and the demands such that some criterion is optimized. The design problem can be formulated as an MPEC, where the traffic equilibrium is described by the system (3) of mixed complementarity constraints. An example of a network design problem is given by setting link tolls through the design parameter $x \in \mathbb{R}^{n}$, with $n \leq|\mathcal{E}|$, such that the total travel cost $f(x, v):=\sum_{l \in \mathcal{E}} t_{l}(x, v) v_{l}$ is minimized, and where, for a given design $x, v$ is given by (3) and (4).

The traffic equilibrium model is a static model. All quantities are assumed to be an average over a time period, and as such they are subjected to uncertainties. The travel 
costs $t(x, v, \omega)$ and demands $d(x, \pi, \omega)$ are to some extent uncertain and can change depending on external factors, such as the weather. Therefore, we can and should formulate the network design problem as an SMPEC, which gives us a design which is the best possible on average. This problem has been studied in Patriksson [6,8]. Birbil et al. [14] consider a similar model in which, however, the response variables are not stochastic, but are solutions to a stochastic equilibrium problem.

For further references on traffic equilibrium models, see $[13,15,16]$.

We present a small numerical example in the application of network design under user equilibrium. The deterministic example is known as Braess' paradox (see e.g. [15, page 75]). It demonstrates that adding an extra link to a network can cause an increase in the total travel cost. In short, this is due to the fact that user equilibrium is a selfish optimum and not a system optimum. Figures 1(a) and 1(b) show the network graph with four and five links, which we will refer to as graph I and graph II, respectively.

We have one OD-pair $(A, B)$ with a fixed demand of $d=6$ units. The original network has two paths, using the links $(1,4)$ and $(3,2)$, respectively; network II has three paths, using the links $(1,4),(3,2)$ and $(3,5,4)$, respectively. The link travel costs are $t_{i}=50+v_{i}$ for $i=1,2, t_{i}=10 v_{i}$ for $i=3,4$, and $t_{5}=10+v_{5}$. Given theses costs, the user equilibrium flows for network I are $v=(3,3,3,3)^{\mathrm{T}}, h=(3,3)^{\mathrm{T}}$. These flows give the equilibrium travel cost $\pi=83$. For network II, the user equilibrium flows are $v=(2,2,4,4,2)^{\mathrm{T}}$, $h=(2,2,2)^{\mathrm{T}}$. These flows give the equilibrium travel cost $\pi=92$. Note that adding a link to network I yields an increase in the equilibrium travel cost.

The idea is to set tolls on network II such that we minimize the total travel cost $T(x, v)=\sum_{i=1}^{5} t_{i} v_{i}$. For the example above, we consider adding a toll $x$ on the new link, thus altering the travel cost to $t_{5}=10+v_{5}+x$, and consider the problem

$$
\begin{array}{cl}
\min _{(x, v, \pi, h)} & T(x, v)+\tau x^{2}, \\
\text { s.t. } & x \in X, \\
& (v, \pi, h) \text { solves (3) and (4), }
\end{array}
$$

where $X=\{x \in \mathbb{R} \mid 0 \leq x \leq 14\}$ and $\tau>0$ is a penalty parameter against setting a too high toll value. For a sufficiently small value of $\tau$, the optimal solution is $x^{*}=13$ and the optimal total travel cost is $T\left(x^{*}, v^{*}\right)=498$. The optimal solution $x^{*}=13$ is the threshold value for which there will be no flow on link 5 , which in turn will give a lower total travel cost.

Now, consider the case when the travel costs are stochastic. In particular, let us assume that the travel costs on links 3 and 4 are

$$
t_{i}=10 v_{i}+\omega_{i-2}, \quad i=3,4,
$$

and that each component in $\omega$ is independent and drawn from a normal distribution with mean 0 and variance 1 , i.e., $\omega \sim \mathcal{N}(0, \operatorname{diag}(1,1))$. We consider the following SMPEC 
model:

$$
\begin{array}{cll}
\min _{(x, v(\cdot), \pi(\cdot), h(\cdot))} & \mathrm{E}_{\omega}[f(x, v(\omega))]:=\mathrm{E}_{\omega}[T(x, v(\omega))]+\tau x^{2}, & \\
\text { s.t. } & x \in X, & \\
& 0 \leq h(\omega) \perp \Lambda^{\mathrm{T}} t(x, v(\omega))-\Gamma \pi(\omega) \geq 0, & P \text {-a.s., } \\
& \Gamma^{\mathrm{T}} h(\omega)=d, & P \text {-a.s., } \\
& v(\omega)=\Lambda h(\omega), & P \text {-a.s., }
\end{array}
$$

where $\Lambda$ and $\Gamma$ are the route-link incidence matrix and the route-OD pair incidence matrix, respectively, for network II. The SMPEC is solved using the discretization scheme SAA. Since the travel costs $t(x, \cdot)$ are strongly monotone, the SMPEC satisfies Assumptions $\mathrm{A}-\mathrm{C}$, and this implies that the optimal solution is stable in the sense of Theorems 2.1 and 2.2 (see also [8]) and that SAA converges by Theorems 2.3 and 2.4 .

Note that the number of variables and constraints scale linearly with the number of scenarios, since we use a general nonlinear optimization solver. This means that, for 100 scenarios, the problem has 900 variables. So, even though the deterministic problem is of small scale, the stochastic version is of large scale. By switching to an implicit function $v(x, \omega)$ and using sensitivity analysis ( [17]), we may be able to solve larger problems. The traffic model was implemented in MATLAB and solved using the solver SNOPT [18].

For a run with $N=400$, the solver converged to the stationary solution $x^{*}=14$. In Figure 2, we plot histograms of the objective values for stationary solutions to three models: one with the expected value in the objective $\left(x^{*}=14.0\right)$, one with $\mathrm{CVaR}$ at $\beta=0.8\left(x^{*}=11.6\right)$ and one with CVaR at $\beta=0.95\left(x^{*}=10.6\right)$.

In order to illustrate the influence of the variance of the uncertain parameter on the solution, we show in Figure 3 histograms of the equilibrium path travel cost for stationary solutions corresponding to four values of the variance of the stochastic variable. The results are not surprising: a larger variance implies a larger spread in the response. (We note in passing that Gwinner and Raciti [19] consider the stochastic traffic network equilibrium model, i.e., the lower-level problem in the SMPEC model, and have developed a procedure for the analytical computation of the mean equilibrium flows and their variance for the case when the travel costs are affine in the flow variables.) Having access to histograms for responses, i.e., equilibrium solutions, is a feature of SMPEC which may be valuable for getting specific insights into an application.

Regarding the solution of the discretized model, we have made the natural observation that the solution time increases with the number of scenarios and that it also increases with the variance of the stochastic variable. Obviously, these observations are based on a single test case and should not be considered as general conclusions.

The design and implementation of a pricing scheme must be simple and transparent; it must also address social welfare issues such as the welfare effect of tolls across population groups. In [20-22], several equity measures are presented and evaluated in the context of optimal network design. In the first two papers, these design models are built upon stochastic (in fact, probit) traffic equilibrium models; such equilibrium solutions are still deterministic functions of the data of the traffic network. The results of our accompanying paper [7], as outlined in the previous section, are immediately transferable to such a setting; see also the discussion sections in [6,8].

Regardless of whether the traffic model is a deterministic or a stochastic equilibrium 
one, the theoretical results of our accompanying paper [7] may provide ideas for several interesting developments. First, we may theoretically validate and numerically study SMPEC versions of network design problems where equity is included as an objective. In the previous papers [20-22] equity measures are treated through upper-level constraints, which however in general result in unstable optimal and stationary solutions. Our proposal is to instead study a bicriterion version of the problem (see the early reference [23] on multiobjective traffic network design), where the original toll efficiency objective is complemented by an equity objective. Moreover, as network user responses in the SMPEC model are stochastic, the equity objective is natural to include as a risk measure.

While link tolling is a decentralized pricing mechanism, signal timings are centralized mechanisms for controlling traffic flows; optimizing signal controls subject to traffic equilibrium constraints is nearly as old a scientific subject as is toll optimization; see e.g. the reviews in $[24,25]$. It is frequently recognized that the performance of signal timings is unstable due to fluctuating traffic conditions, such as fluctuating demands (see e.g. [26]). The above example serves to illustrate the potential in utilizing the SMPEC formalism in this field.

\section{Second Application: Optimization of a Treatment Plan for Radiation Therapy}

In radiation therapy, cancerous tumors are subjected to ionizing radiation. The objective is to eradicate the tumor while sparing the surrounding tissue and organs at risk. We will show that MPEC models can be utilized to find optimal radiation plans.

Radiation is delivered by a linear accelerator and, by using what is called multileaf collimators, the radiation beam can be shaped such that different parts in the treatment region receive different doses. This technique of shaping the beam is called intensity modulated radiation therapy (IMRT). Since there are millions of ways of modulating the intensity, the most suitable radiation dose is found by optimization. The ideal dose is still often not attainable, so the objective is to find the best compromise achievable.

The linear accelerator can deliver radiation to the target from several angles by the use of a gantry arm. For some cases, up to nine gantry angles are used to give a good target coverage. The angles are usually considered fixed in the optimization problem.

We will describe two methods for parameterizing the multileaf collimator system. Both methods may act as the lower-level problem in an (S)MPEC setting. The beam crosssection is subdivided into small rectangular cells, known as beamlets, and the decision variables are the intensities through each cell. In the first method, we assume that any bounded, nonnegative, intensity profile is attainable by multileaf collimators as a total over a treatment time. We also assume that the doses scale linearly with the intensities and are additive.

In the other method, the leaf trajectories are parameterized; given a desired intensity, the trajectories are found through an optimization problem.

Objective functions in IMRT are either physically or biologically based. In short, a physically based function is a function of the dose alone. It can, for example, be the quadratic deviation from a dose level sought or the maximum dose in a domain. A 
biologically based function is associated with a specific organ and measures the biological effect of a dose. The function is constructed using a set of biological parameters which can depend on the organ type, its size, shape etc.

An example of a biologically based function is the normal tissue complication probability (NTCP) (see e.g. [27]):

$$
\mathrm{NTCP}=\frac{1}{\sqrt{2 \pi}} \int_{-\infty}^{u} \exp ^{-t^{2} / 2} d t,
$$

where

$$
u=\frac{\operatorname{GEUD}(d)-D_{50}}{m D_{50}},
$$

and $D_{50}$ is the homogeneous dose corresponding to $50 \%$ risk of complication, $m$ determines the slope of the risk, $d$ is the dose, and GEUD ( [28]) is the generalized equivalent uniform dose,

$$
\operatorname{GEUD}(d)=\left(\frac{1}{|J|} \sum_{j \in J} d_{i}^{a}\right)^{1 / a},
$$

where $J$ is the set of voxels (discretized cells) in the organ. The parameter $a$ influences the volume effect of the dose: if $a=1$, then the function measures the mean dose; for higher values, the function value approaches the maximum dose. For example, the spinal cord is sensitive to a maximum dose and the volume effect is low. On the other hand, the parotid glands are organs that are sensitive to how much volume receives a certain dose. Suitable values for $a$ are typically fit from clinical data. The GEUD function may be used as an objective function by itself. The function is attractive from several points of view, not the least the fact that it is convex if $a \geq 1$, which is the case for organs.

Radiation therapy is delivered in fractions over several weeks; although the patient is fixated, there will be variations in position over the sessions. (This is called setup errors.) Another uncertainty that affects how good a treatment plan is in practice is the patient and organ motion which will vary during a treatment session. Combining the two, we get a position uncertainty. Olafsson and Wright [29] and Chu et al. [30] assume that the doses are stochastic and use probabilistic constraints to control the dose levels in the target and in the organs at risk. Chan et al. [31] use a motion probability mass function and assumes that the probability itself is uncertain. Using linear programming duality, they can formulate the optimization problem as a large linear program. Baum et al. [32] use coverage probabilities for the target and tumor as penalties in the objective functions to derive a robust treatment. Unkelbach and Oelfke [33] discuss, from a mathematical and a physics perspective, the difference between using coverage probabilities and stochastic programming in IMRT optimization.

Biological uncertainty can also be incorporated in an SMPEC model through the objective function. An optimal solution then is the best from a population perspective. Functions based on the biological effect have certain advantages over physically based ones, but they rely on the accuracy of biological parameters which are fit from data in medical studies. For example, the dose-volume effect for the bladder is uncertain (see e.g. $[34,35])$, which has an impact on the parameter $a$ in the GEUD function. 
In Kåver et al. [36] and Lian and Xing [37], stochastic programming is used for the optimization of a treatment plan when there are uncertainties in the biological parameters. In both papers, the expected value of the objective functions is minimized. Kåver et al. use the objective $P_{+}$( [38]), which is a nonconvex objective function; Lian and Xing use an objective function based on GEUD. Lian and Xing report that the result strongly depends on the underlying probability distribution.

For more general information on IMRT, see e.g. [39-41].

\subsection{Linear Lower-Level Problem}

Let $y \in \mathbb{R}^{m}$ denote the dose in the voxels and let $x \in X$ denote the intensities (beamlets) in the beam. If the dose scales linearly and is additive, an attainable dose is given by the equation

$$
y=K x,
$$

where the influence matrix $K \in \mathbb{R}^{m \times n}$ is computed beforehand. To put this parameterization into the (S)MPEC framework, let $x$ denote the decision variables and let $y$ denote the response variables. The equation (6) then represents the lower-level problem.

In connection with this setting, we consider a prostate case where the PTV (Planned Target Volume) overlaps two critical structures: the rectum and the bladder (see Figure 4). We have four objectives, which are listed in Table 1. The PTV should receive a uniform dose of $d^{P}=70 \mathrm{~Gy}(\mathrm{~J} / \mathrm{kg})$. The rectum is considered as an organ with a serial architecture, which means that it is sensitive to the maximum dose. The volume effect parameter for this organ is set to $a_{r}=8.3$ according to Emami et al. [42]. The architecture of the bladder is more uncertain, but it is set to $a_{b}=2$ [42].

In Table 1, the structure 'Unspecified' refers to the normal tissue surrounding the other structures. We enforce a maximum dose limit $d^{N}=50 \mathrm{~Gy}$ on this structure. This is quite common in clinical practice in order to avoid hot spots which can induce secondary cancers.

The upper limits for the GEUD for the rectum $g_{r}$ and for the bladder $g_{b}$ were computed from the treatment plan which was used in the clinic for this patient.

Since the structures overlap, the four objectives will be in conflict with each other; it is our goal to find a good compromise. This is a multiple objective problem for which Theorem 2.5 and 2.6 represent the stability results obtainable. We use a simple scalarization of the objective functions.

Target coverage is enforced by minimizing a quadratic measure of the deviation. We assume that the location of the tumor is uncertain and consider it to move like a rigid object with a radial offset. We choose to ignore any fractionation effect and consider the treatment to be given at a single session. This means that we assume that the total dose $D$ for the target after $N$ fractions is $D(\omega)=\sum_{i=1}^{N} d\left(\omega^{i}\right)$; it is assumed to have its center normally distributed with standard deviation $0.6 \mathrm{~cm}$. Furthermore, let $\mathcal{T}(\omega), \mathcal{B}, \mathcal{R}, \mathcal{N}$ denote the voxels in the PTV, the bladder, the rectum and the normal tissue, respectively. For a vector $v \in \mathbb{R}^{n}$ and a set $I=\left\{i_{1}, \ldots, i_{k}\right\}$ with $|I| \leq n$, we use the notation

$$
v_{I}=\left(v_{i_{1}}, \ldots, v_{i_{k}}\right)^{\mathrm{T}} \quad \text { and } \quad v_{+}=\left(\max \left\{0, v_{1}\right\}, \ldots, \max \left\{0, v_{n}\right\}\right)^{\mathrm{T}} .
$$

Also, we let $e=(1, \ldots, 1)^{\mathrm{T}}$. 
Consider the following multiobjective problem, which is of the form $\left(\mathrm{SMOPEC}_{\Omega}\right)$ :

$$
\begin{array}{ll}
\left(\operatorname{SIMRT}_{\Omega}\right) & \min _{(x, d)} \\
& \left(\mathrm{E}_{\omega}\left[Q_{1}(d, \omega)\right], Q_{2}(d)\right), \\
& \text { s.t. } \quad x_{j} \in X_{j}, \quad j=1, \ldots, n, \\
& d=K x,
\end{array}
$$

where $X_{j}=\left[0, u_{j}\right]$ are lower and upper bounds on the intensities and the objective functions are defined as

$$
\begin{aligned}
Q_{1}(d, \omega):= & \frac{1}{2|\mathcal{T}(\omega)|}\left(d_{\mathcal{T}(\omega)}-s_{0}\right)^{\mathrm{T}} S_{0}^{-1}\left(d_{\mathcal{T}(\omega)}-s_{0}\right), \\
Q_{2}(d):= & \frac{1}{g_{r}}\left(\frac{1}{|\mathcal{R}|} \sum_{j \in \mathcal{R}} d_{j}^{a_{r}}\right)^{1 / a_{r}}+\frac{1}{g_{b}}\left(\frac{1}{|\mathcal{B}|} \sum_{j \in \mathcal{B}} d_{j}^{a_{b}}\right)^{1 / a_{b}} \\
& +\frac{1}{2|\mathcal{N}|}\left(d_{\mathcal{N}}-r_{0}\right)_{+}^{\mathrm{T}} R_{0}^{-1}\left(d_{\mathcal{N}}-r_{0}\right)_{+},
\end{aligned}
$$

where

$$
s_{0}=d^{P} e, S_{0}=\operatorname{diag}\left(s_{0}\right), r_{0}=d^{N} e, R_{0}=\operatorname{diag}\left(r_{0}\right) .
$$

Both $Q_{1}$ and $Q_{2}$ are convex functions; hence, $\left(\mathrm{SIMRT}_{\Omega}\right)$ is a convex problem. The function $Q_{1}$ measures the deviation from the target dose $d^{P} ; Q_{2}$ is based on the GEUD functions for the rectum and the bladder, respectively, and a one-sided measure of the deviation from the maximum dose in the unspecified tissue.

The uncertainty in $\left(\mathrm{SIMRT}_{\Omega}\right)$ enters only into the objective functions. The lower-level problem is the trivial linear equation, $d=K x$, and so the assumptions of Theorem 2.5 are fulfilled: weakly Pareto-optimal solutions are stable.

We use beams from 5 equidistant gantry angles to irradiate the tumor. The number of voxels and variables in $\left(\mathrm{SIMRT}_{\Omega}\right)$ depends on the number of beams, the beamlet size and the geometry and resolution of the patient region. For this case, we have 1,526,330 voxels and $n=336$ variables. We let $u_{j}=30$ for $j=1, \ldots, n$. The IMRT model was implemented in Fortran 90. The radiation treatment planning tool CERR [43] was used to setup the problem, and LANCELOT B [44] was used as the optimization solver.

We compare three models: a conventional treatment where we use a static target with a $1 \mathrm{~cm}$ extra margin $\left(\mathcal{T}(\omega)\right.$ in $\left(\operatorname{SIMRT}_{\Omega}\right)$ is replaced by a static set); a treatment with $\left(\operatorname{SIMRT}_{\Omega}\right)$; and a treatment with $\left(\mathrm{SIMRT}_{\Omega}\right)$ but with conditional value-at-risk at level $\beta=0.8$ instead of the expected value. We compute only one solution on the Pareto surface by setting the constraint $Q_{1} \leq 0$; see Figure 5 . Using an extra margin is the most conservative choice as it will "guarantee" that the tumor gets a sufficient dose, although at the expense of extra radiation to the risk organs. The expected value is the least conservative choice, as it constrains the tumor in a mean sense; the CVaR objective is a compromise. The conventional treatment gives a $2.7 \%$ risk of rectal bleeding $\left(m=0.15, D_{50}=80 \mathrm{~Gy}[42]\right)$, while the expected value results in the risk being reduced to $0.9 \%$. If CVaR at level $\beta=0.8$ is used, the risk is $1.1 \%$.

The results show that, if there is a willingness to be less conservative in the choice of target coverage, then there is a benefit in using stochastic models in terms of risk of complications. 


\subsection{Deliverable Treatment}

The multileaf collimators (MLC) that shape the beam are organized as pairs. If we consider the rectangular beam cross-section as a matrix, where each element represents a discretized cell, then each leaf pair can block a "column"; see Figure 6 (note that, in the figure, each leaf blocks one row). Let $I=\{1, \ldots, m\}, J=\{1, \ldots, n\}$, and let $x_{i j}, j \in J$, $i \in I$, be the desired intensity (decision variables) in beamlet $(i, j)$. We assume that there are $n$ pairs of MLC leaves $\left(A_{j}, B_{j}\right), j \in J$. We assume that the leaves move from row 1 to row $m$.

Let $a_{i j}$ and $b_{i j}$ denote the cumulative beam-on time in monitor units of leaf $A_{j}$ and leaf $B_{j}$, respectively, at row $i$. Assuming that each leaf totally blocks the radiation, and that it "jumps" instantaneously from row $i$ to row $i+1$, the delivered intensity $y$ satisfies $y_{i j}=a_{i j}-b_{i j}$. The objective with leaf motion computation is to determine the beam-on time $a$ and $b$ such that the desired intensity is obtained, in minimum total time (beam-on time).

The leaves are constrained to not exceed a maximum speed, which implies that there is a minimum time difference between the beam-on time for two consecutive rows. If we assume that the distance between all rows are constant, we can set the minimum time difference to $\Delta t$.

We assume that each pair of leaves begin at row 1 . In reality, leaf $B$ could be positioned at $i>1$ if the intensities permit it. This would reduce the total time.

Since the beam must be on until the slowest leaf pair finishes, the leaves $A_{j}$ are also constrained to end at row $m$ at the same time. This would avoid any extra radiation through the unclosed leaves. The optimization problem to deliver an intensity $x$, in minimum time, is formulated as:

$$
\begin{aligned}
& \text { (TLP) } \min _{(t, a, b)} t \\
& \text { s.t. } \quad a_{i+1 j} \geq a_{i j}+\Delta t, \quad i=1, \ldots, m-1 \text {, } \\
& b_{i+1 j} \geq b_{i j}+\Delta t, \quad i=1, \ldots, m-1, \\
& a_{m, j}=t, \quad j \in J, \\
& x_{i j}=a_{i j}-b_{i j}, \quad i \in I, j \in J, \\
& a_{i j} \geq 0, \quad i \in I, j \in J, \\
& b_{i j} \geq 0, \quad i \in I, j \in J .
\end{aligned}
$$

This model was formulated by Convery and Rosebloom [45]. Spirou and Chui [46] present an analytic expression for the optimal solution, which is derived from the fact that one of the leaves in each pair must move at the maximum speed. Consider the pair $j$ and assume that $a_{i j}$ and $b_{i j}$ are known.

$$
\begin{array}{ll}
\text { If } x_{i+1 j} \geq x_{i j} \text {, then } & \left\{\begin{array}{l}
b_{i+1 j}=b_{i j}+\Delta t, \\
a_{i+1 j}=b_{i+1 j}+x_{i+1 j},
\end{array}\right. \\
\text { If } x_{i+1 j}<x_{i j} \text {, then } & \left\{\begin{array}{l}
a_{i+1 j}=a_{i j}+\Delta t, \\
b_{i+1 j}=a_{i+1 j}-x_{i+1 j} .
\end{array}\right.
\end{array}
$$


In reality, there are phenomena which effect the delivered intensity $y$. One such is leaf transmission, which is considered by Spirou and Chui [46]. Let $\tau$ be the leaf transmission factor and let $t^{*}$ be the total beam-on time. Then the intensity delivered is

$$
y_{i j}=\tau\left[t^{*}-\left(a_{i j}-b_{i j}\right)\right]+a_{i j}-b_{i j}
$$

where $t^{*}-\left(a_{i j}-b_{i j}\right)$ is the total time cell $(i, j)$ is blocked and there is a transmission. From the analytic expressions (7), the total beam-on time is given by ( $[46])$

$$
t^{*}=\max _{j}\left(n \Delta t+x_{m j}+\sum_{i=1}^{m-1}\left[x_{i+1 j}-x_{i j}\right]_{+}\right) .
$$

We consider the linear optimization problem (TLP) as the lower-level problem in an (S)MPEC framework. We let $x$ denote the decision variables. If we let the total beamon time $t$ denote the response variable ( $a$ and $b$ are eliminated in (8) by the equation $x_{i j}=a_{i j}-b_{i j}$ ), we see that, since it is Lipschitz continuous, it fulfills the requirements on the lower-level problem for global and stationary stability, cf. Theorem 2.1 and 2.2. On the other hand, if we let $a$ and $b$ denote the response variables, we note that they are not Lipschitz continuous with respect to $x$ as is shown in the analytic expressions. However, in practice, if we consider a nonoptimal heuristic solution in the lower-level problem, we can modify the analytic expressions with a ramp to make the responses $A$ and $B$ Lipschitz continuous with respect to $x$, and this will give stability.

We now consider the same prostate case as in the previous subsection, with the exception that the dose to the tumor should be $\pm 3 \%$. We assume that the locations are static, but the leaf trajectories $A$ and $B$ are stochastic. This is manifested in that, at each position $(i, j)$, there can be a delay in the beam-on time, which corresponds to the fact that the leaves may unexpectedly move more slowly than anticipated. This is to some extent observed in reality ( $[47]$ ). Consider the following stochastic multiobjective problem:

$$
\begin{aligned}
& \left(\operatorname{SDEL}_{\Omega}\right) \quad \min _{(x, y(\cdot), a(\cdot), b(\cdot), t(\cdot))}\left(\mathrm{E}_{\omega}\left[G_{1}(d(\omega))\right], \mathrm{E}_{\omega}\left[G_{2}(d(\omega))\right]\right), \\
& \text { s.t. } \quad x \in X \text {, } \\
& y(\omega)=\tau(t-(a-b)) \\
& +(1+c(\omega))(a-b), \quad P \text {-a.s., } \\
& d(\omega)=K y(\omega), \quad P \text {-a.s., } \\
& (a, b, t) \text { solves (TLP) given } x \text {, }
\end{aligned}
$$

where $c$ determines the speed lag and

$$
\begin{aligned}
G_{1}(d(\omega)):= & \frac{1}{2|\mathcal{T}|}\left(d_{\mathcal{T}}-s_{0}\right)^{\mathrm{T}} S_{0}^{-1}\left(d_{\mathcal{T}}-s_{0}\right) \\
G_{2}(d(\omega)):= & \frac{1}{g_{r}}\left(\frac{1}{|\mathcal{R}|} \sum_{j \in \mathcal{R}} d_{j}^{a_{r}}\right)^{1 / a_{r}}+\frac{1}{g_{b}}\left(\frac{1}{|\mathcal{B}|} \sum_{j \in \mathcal{B}} d_{j}^{a_{b}}\right)^{1 / a_{b}} \\
& +\frac{1}{2|\mathcal{N}|}\left(d_{\mathcal{N}}-r_{0}\right)_{+}^{\mathrm{T}} R_{0}^{-1}\left(d_{\mathcal{N}}-r_{0}\right)_{+}
\end{aligned}
$$


The function $c$ is chosen such that, for each beam, $10 \%$ of the positions are affected. Let $k \in \mathcal{K}$ denote the affected intensities, let $\omega_{k} \sim \mathcal{N}(0.1,0.1) \cap \mathbb{R}_{+}$for $k \in \mathcal{K}$, let $c_{k}=\omega_{k}$ for $k \in K$, and let $c_{k}=0$ for $k \notin K$. The multiobjective problem is discretized using SAA with 20 samples and solved using the epsilon-constrained method ( [48]). The model was implemented in Fortran 90 and LANCELOT B [44] was used as the optimization solver. The lower-level problem was implemented as an implicit function.

Figure 7 shows the local Pareto front for solutions to $\mathrm{SDEL}_{\Omega}$. As a comparison, locally optimal solutions $x^{*}$, corresponding to the deterministic plan $(c \equiv 0)$, are evaluated in $\mathrm{SDEL}_{\Omega}$. The results show that there is an unexpected leakage of radiation due to slower moving leaves, and this in turn implies that the organs receive an additional dose. The SMPEC model is to some extent able to cope with this situation and delivers on average a better treatment plan compared to a deterministic model.

\section{Summary, Conclusions and Future Research}

This paper contributes with two rather different applications of robust SMPEC models.

First, we consider the case of robust network design under tolled user equilibrium flows. This is a case where the lower-level problem is a variational inequality over a polyhedral set, and where uncertainty may be present in both the demand and travel cost functions. Our numerical example focuses on the latter, and we experiment with both the traditional average and the CVaR objective. Robustness of stationary solutions follows, since we assume the travel cost to be separable and affine with positive coefficients. In practice, link costs normally are modeled as nonlinear. In order to establish the robustness of stationary solutions and the convergence of Monte Carlo schemes, Assumption (B) must be enforced (cf. Theorems 2.2 and 2.4), which implies a strong monotonicity assumption on the travel cost; this may be a limiting factor in some applications. A particularly nice feature of the present application is the availability of information from the responses generated in the Monte Carlo scheme, as revealed in Figures 2 and 3. In applications of toll optimization, where equity is an important issue, the CVaR objective provides a very interesting performance measure, as it allows for the optimization of the worst case situation. It would be interesting to further study applications of robust toll setting problems under uncertainty in this setting, in particular in the multiclass, elastic demand setting.

Second, we consider the optimization of a robust treatment plan in intensity modulated radiation therapy. We consider two lower-level problems, each with its unique type of uncertainty: one with a simple linear system of equations, where the position of the tumor is uncertain, and one with a linear program where some of the machine parameters are uncertain. In the first of these examples, both the expected value and the CVaR objective are used to tackle the position uncertainty. The properties of the simple lowerlevel problem imply that the problem is convex and also that globally optimal solutions are robust. The numerical example shows that the risk of complications can be reduced significantly if the conservative extra margin around the tumor is reduced and tumor coverage is enforced with expected value or CVaR minimization.

The linear program in the lower-level problem represents the problem to find optimal 
leaf trajectories to block the beam such that a given intensity profile is (almost) attained in minimum time. Compared to the other version with a linear lower-level problem, this formulation is approaching what is called a deliverable treatment. This means that the optimal solution can be used with much less postprocessing, which may otherwise worsen the plan. We assume that the speed of the leaves are uncertain, which implies that there will be unexpected leakages of radiation. Due to the presence of a linear lowerlevel problem, the resulting SMPEC violates Assumption (B); to establish robustness, we consider a heuristic solution to the lower-level problem. We consider the optimization of a treatment plan as a multiobjective problem with two goals: tumor coverage and minimum risk of complications. It was found that the uncertainty in general reduces the quality of a plan due to the radiation leakage, but that the SMPEC model yields a better treatment plan on average compared to a deterministic model.

We already utilize biological objective functions in our IMRT application, and they are becoming increasingly important in practice. As a future research task, it would be interesting to consider uncertainty also in the radiobiological parameters. Their inclusion into the SMPEC model should be straightforward.

For the application of the SMPEC model to become more practical, special algorithms need to developed.

Acknowledgements This work was financially partially supported by the Gothenburg Mathematical Modeling Centre (GMMC) at Mathematical Sciences (Chalmers University of Technology and the University of Gothenburg), whose main sponsor is the Swedish Foundation for Strategic Research (SSF). The authors wish to thank Caroline Olsson for valuable help with the biology of radiation therapy and for providing a test case. We finally thank the Associate Editor Franco Giannessi for his very encouraging and constructive remarks on a previous manuscript.

\section{References}

[1] Patriksson, M., Wynter, L.: Stochastic mathematical programs with equilibrium constraints. Oper. Res. Lett. 25(4), 159-167 (1999)

[2] Couchman, P., Kouvaritakis, B., Cannon, M., Prashad, F.: Gaming strategy for electric power with random demand. IEEE Trans. Power Syst. 20(3), 1283-1292 (2005)

[3] Xu, H.: An MPCC approach for stochastic Stackelberg-Nash-Cournot equilibrium. Optimization 54(1), 27-57 (2005)

[4] Christiansen, S., Patriksson, M., Wynter, L.: Stochastic bilevel programming in structural optimization. Struct. Multidiscip. Optim. 21(5), 361-371 (2001)

[5] Evgrafov, A., Patriksson, M., Petersson, J.: Stochastic structural topology optimization: existence of solutions and sensitivity analyses. ZAMM Z. Angew. Math. Mech. 83(7), 479-492 (2003) 
[6] Patriksson, M.: Robust bi-level optimization models in transportation science. Philos. Trans. R. Soc. Lond. Ser. A Math. Phys. Eng. Sci. 366(1872), 1989-2004 (2008)

[7] Cromvik, C., Patriksson, M.: On the robustness of global optima and stationary solutions to stochastic mathematical programs with equilibrium constraints, part I: Theory. J. Optim. Theory Appl. (2009, to appear)

[8] Patriksson, M.: On the applicability and solution of bilevel optimization models in transportation science: A study on the existence, stability and computation of optimal solutions to stochastic mathematical programs with equilibrium constraints. Transp. Res. 42B(10), 843-860 (2008)

[9] Evgrafov, A., Patriksson, M.: On the existence of solutions to stochastic mathematical programs with equilibrium constraints. J. Optim. Theory Appl. 121(1), 65-76 (2004)

[10] Robinson, S.M.: Strongly regular generalized equations. Math. Oper. Res. 5(1), 43-62 (1980)

[11] Wardrop, J.G.: Some theoretical aspects of road traffic research. In: Proceedings of the Institute of Civil Engineers, Part II, pp. 325-378 (1952)

[12] Aashtiani, H.Z., Magnanti, T.L.: Equilibria on a congested transportation network. SIAM J. Algebraic Discrete Methods 2(3), 213-226 (1981)

[13] Patriksson, M.: The Traffic Assignment Problem: Models and Methods. VSP, Zeist (1994)

[14] Birbil, Ş.İ., Gürkan, G., Listeş, O.: Solving stochastic mathematical programs with complementarity constraints using simulation. Math. Oper. Res. 31(4), 739-760 (2006)

[15] Sheffi, Y.: Urban Transportation Networks. Prentice-Hall, Englewood Cliffs, NJ (1985)

[16] Marcotte, P., Patriksson, M.: Traffic equilibrium. In: C. Barnhart, G. Laporte (eds.) Transportation, Handbooks in Operations Research and Management Science, vol. 14, pp. 623-713. North-Holland, Amsterdam (2007)

[17] Sensitivity analysis of separable traffic equilibrium equilibria with application to bilevel optimization in network design. Transp. Res. 41B(1), 4-31 (2007)

[18] Gill, P.E., Murray, W., Saunders, M.A.: SNOPT: an SQP algorithm for large-scale constrained optimization. SIAM Rev. 47(1), 99-131 (2005)

[19] Gwinner, J., Raciti, F.: Random equilibrium problems on networks. Math. Comput. Modelling 43(7-8), 880-891 (2006)

[20] Sumalee, A.: Optimal toll ring design with spatial equity impact constraint: An evolutionary approach. J. East. Asia Soc. Transp. Stud. 5, 1813-1828 (2003) 
[21] Connors, R., Sumalee, A., Watling, D.: Equitable network design. J. East. Asia Soc. Transp. Stud. 6, 1382-1397 (2005)

[22] Maruyama, T., Sumalee, A.: Efficiency and equity comparison of cordon- and areabased road pricing schemes using a trip-chain equilibrium model. Transp. Res. 41A(7), 655-671 (2007)

[23] Friesz, T.L., Anandalingam, G., Mehta, N.J., Nam, K., Shah, S.J., Tobin, R.L.: The multiobjective equilibrium network design problem revisited: A simulated annealing approach. European J. Oper. Res. 65(1), 44-57 (1993)

[24] Migdalas, A.: Bilevel programming in traffic planning: Models, methods and challenge. J. Global Optim. 7(4), 381-405 (1995)

[25] Larsson, T., Patriksson, M.: Side constrained traffic equilibrium models - traffic management through link tolls. In: P. Marcotte, S. Nguyen (eds.) Equilibrium and Advanced Transportation Modelling, pp. 125-151. Kluwer Academic Publishers, Boston, MA (1998)

[26] Yin, Y.: Robust optimal traffic signal timing. Transp. Res. 42B(10), 911-924 (2008)

[27] Stavrev, P., Hristov, D., Warkentin, B., Sham, E., Stavreva, N., Fallone, B.G.: Inverse treatment planning by physically constrained minimization of a biological objective function. Med. Phys. 30(11), 2948-2958 (2003)

[28] Niemierko, A.: Reporting and analyzing dose distributions: A concept of equivalent uniform dose. Med. Phys. 24(1), 103-110 (1997)

[29] Olafsson, A., Wright, S.J.: Efficient schemes for robust IMRT treatment planning. Phys. Med. Biol. 51(21), 5621-5642 (2006)

[30] Chu, M., Zinchenko, Y., Henderson, S.G., Sharpe, M.B.: Robust optimization for intensity modulated radiation therapy treatment planning under uncertainty. Phys. Med. Biol. 50(23), 5463-5477 (2005)

[31] Chan, T.C.Y., Bortfeld, T., Tsitsiklis, J.N.: A robust approach to IMRT optimization. Phys. Med. Biol. 51(10), 2567-2583 (2006)

[32] Baum, C., Alber, M., Birkner, M., Nüsslin, F.: Robust treatment planning for intensity modulated radiotherapy of prostate cancer based on coverage probabilities. Radiother. Oncol. 78(1), 27-35 (2006)

[33] Unkelbach, J., Oelfke, U.: Relating two techniques for handling uncertainties in IMRT optimization. Phys. Med. Biol. 51(23), 423-427 (2006)

[34] Nakamura, R.A., Monti, C.R., Castilho, L.N., Trevisan, F.A., Valim, A.C., Reinato, J.A.: Prognostic factors for late urinary toxicity grade 2-3 after conformal radiation therapy on patients with prostate cancer. Int. Braz. J. Urol. 33(5), 652-659 (2007) 
[35] Harsolia, A., Vargas, C., Yan, D., Brabbins, D., Lockman, D., Liang, J., Gustafson, G., Vicini, F., Martinez, A., Kestin, L.L.: Predictors for chronic urinary toxicity after the treatment of prostate cancer with adaptive three-dimensional conformal radiotherapy: dose-volume analysis of a phase II dose-escalation study. Int. J. Radiat. Oncol. Biol. Phys. 69(4), 1100-1109 (2007)

[36] Kåver, G., Lind, B.K., Löf, J., Liander, A., Brahme, A.: Stochastic optimization of intensity modulated radiotherapy to account for uncertainties in patient sensitivity. Phys. Med. Biol. 44(12), 2955-2969 (1999)

[37] Lian, J., Xing, L.: Incorporating model parameter uncertainty into inverse treatment planning. Phys. Med. Biol. 31(9), 2711-2720 (2004)

[38] Brahme, A.: Individualizing cancer treatment: biological optimization models in treatment planning and delivery. Int. J. Radiat. Oncol. Biol. Phys. 49(2), 327-337 (2001)

[39] Webb, S.: Intensity-Modulated Radiation Therapy. Institute of Physics Publishing, London (2001)

[40] Löf, J.: Development of a general framework for optimization of radiation therapy. Ph.D. thesis, Stockholm University, Stockholm (2000)

[41] Carlsson, J.: Utilizing problem structure in optimization of radiation therapy. Ph.D. thesis, Royal Institute of Technology, Stockholm (2008)

[42] Emami, B., Lyman, J., Brown, A., Coia, L., Goitein, M., Munzenrider, J.E., Solin, L.J., Wesson, M.: Tolerance of normal tissue to therapeutic irradiation. Int. J. Radiat. Oncol. Biol. Phys. 21(1), 109-122 (1991)

[43] Deasy, J., Blanco, A.I., Clark, V.H.: CERR: A computational environment for radiotherapy research. Med. Phys. 30(5), 979-985 (2003)

[44] Gould, N.I.M., Orban, D., Toint, P.L.: GALAHAD, a library of thread-safe Fortran 90 packages for large-scale nonlinear optimization. ACM Trans. Math. Software 29(4), 353-372 (2004)

[45] Convery, D.J., Rosebloom, M.E.: The generation of intensity-modulated fields for conformal radiotherapy by dynamic collimation. Phys. Med. Biol. 37(6), 1359-1374 (1992)

[46] Spirou, S.V., Chui, C.S.: Generation of arbitrary intensity profiles by dynamic jaws or collimators. Med. Phys. 21(7), 1031-1041 (1994)

[47] Chui, C.S., Spirou, S., LoSasso, T.: Testing of dynamic multileaf collimation. Med. Phys. 23(5), 635-641 (1996)

[48] Ehrgott, M.: Multicriteria Optimization, second edn. Springer-Verlag, Berlin (2005) 


\section{List of Tables}

1 Treatment parameters. . . . . . . . . . . . . . . . 22 


\begin{tabular}{cc}
\hline Structure & Prescription \\
\hline PTV & Uniform dose of $d^{P}=70 \mathrm{~Gy}( \pm 5 \%)$ \\
Bladder & gEUD $(a=2)$ below $32 \mathrm{~Gy}$ \\
Rectum & gEUD $(a=8.3)$ below $58 \mathrm{~Gy}$ \\
Unspecified & Maximum dose of $d^{N}=50 \mathrm{~Gy}$ \\
\hline
\end{tabular}

Table 1: Treatment parameters. 


\section{List of Figures}

1 Networks I and II. . . . . . . . . . . . . . . . . . . . . . 24

2 Histograms $(N=400)$ for the objective values: one with expected value in the objective, one with $\mathrm{CVaR}$ at $\beta=0.8$, and one with $\mathrm{CVaR}$ at $\beta=0.95 .25$

3 Equilibrium path travel costs for runs with $N=100$ and with variance $\sigma_{j}^{2}=0.01, \sigma_{j}^{2}=0.025, \sigma_{j}^{2}=1$, and $\sigma_{j}^{2}=4, j=1,2$, for the link costs in (5). Each subfigure corresponds to one stationary solution. . . . . . . . . 26

4 Transverse CT scan. . . . . . . . . . . . . . . . . . . 27

5 Dose-volume histograms for the organs at risk: bladder, rectum, and normal tissue. The dashed line corresponds to the conventional treatment (both figures), while the solid line corresponds to the expected value objective (left figure) and the CVaR objective (right figure) . . . . . . . . . . 28

6 Snapshot of the motion of the multileaf collimators (left figure) for the fluence (intensity) profile shown in the right figure for a cross-section of the beam. . . . . . . . . . . . . . . . . . 29

7 The figure shows three fronts: one is a local Pareto front for solutions $x_{s}^{*}$ to the stochastic model $\mathrm{SDEL}_{\Omega}$ (Stoch.); one is a local Pareto front for solutions $x_{d}^{*}$ to the corresponding deterministic model, i.e., with $c \equiv 0$ (Det.); and one is the deterministic solutions $x_{d}^{*}$ evaluated in $\mathrm{SDEL}_{\Omega}$ (Det. with exp.). . . . . . . . . . . . . . . . . . . . 


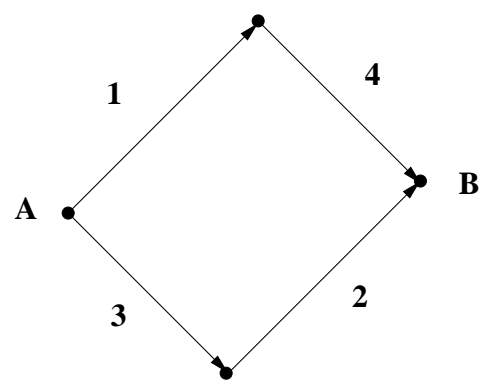

(a) Original network.

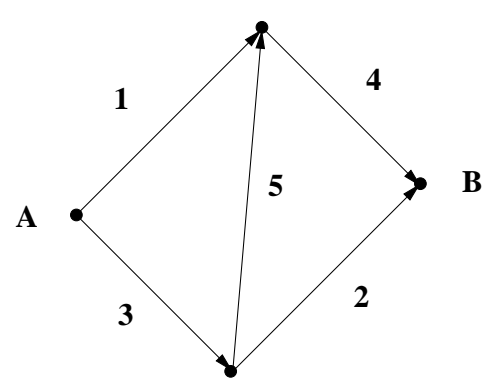

(b) Network with the addition of a new link.

Figure 1: Networks I and II. 

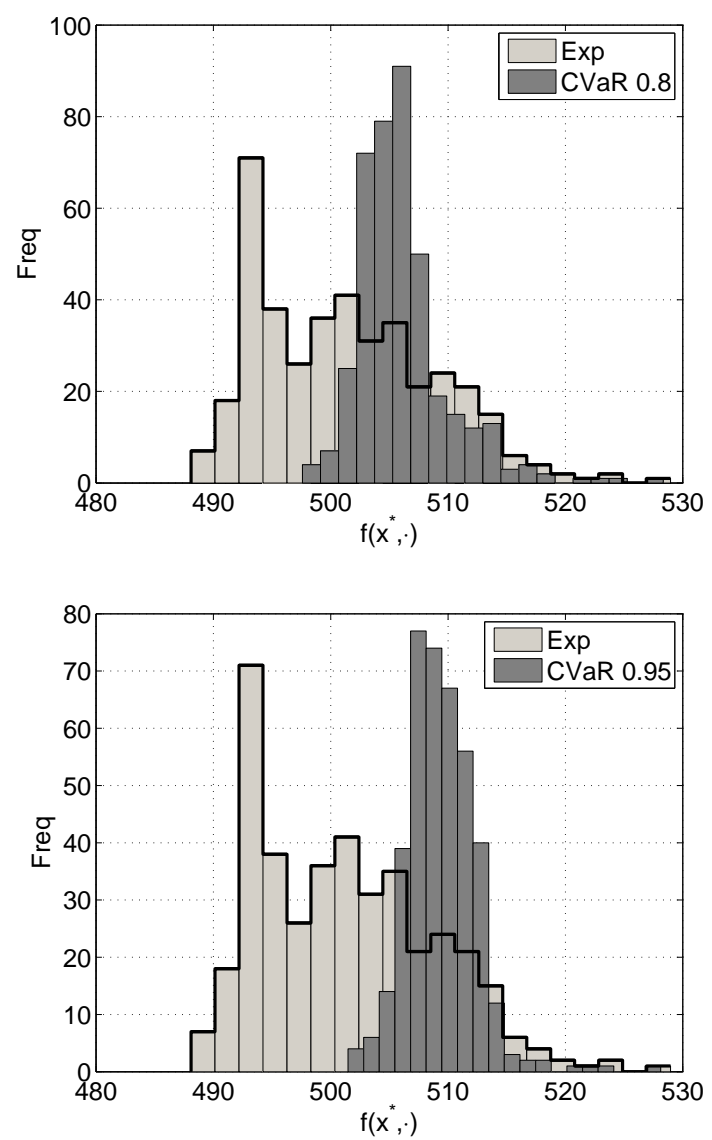

Figure 2: Histograms $(N=400)$ for the objective values: one with expected value in the objective, one with $\mathrm{CVaR}$ at $\beta=0.8$, and one with $\mathrm{CVaR}$ at $\beta=0.95$. 

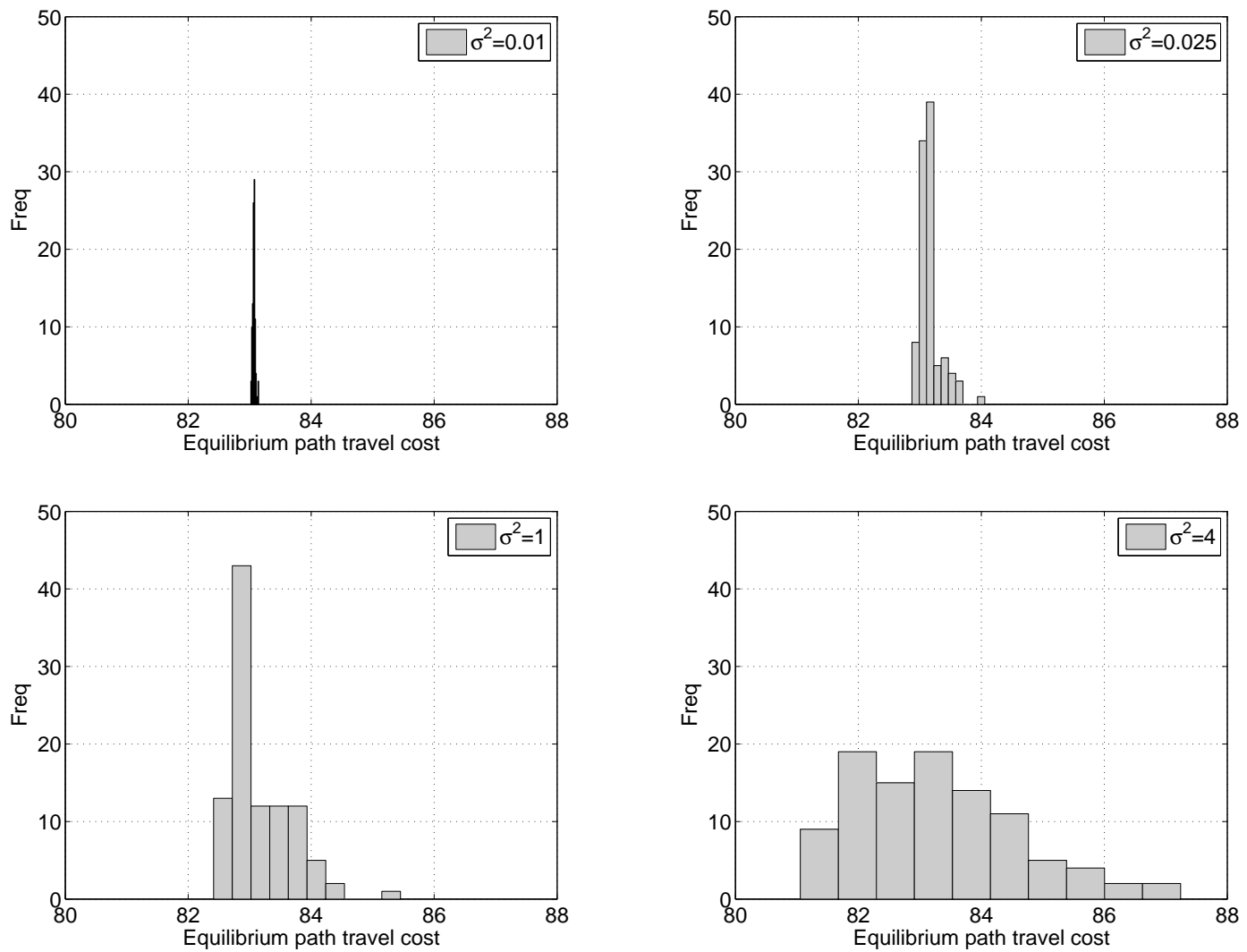

Figure 3: Equilibrium path travel costs for runs with $N=100$ and with variance $\sigma_{j}^{2}=$ $0.01, \sigma_{j}^{2}=0.025, \sigma_{j}^{2}=1$, and $\sigma_{j}^{2}=4, j=1,2$, for the link costs in (5). Each subfigure corresponds to one stationary solution. 


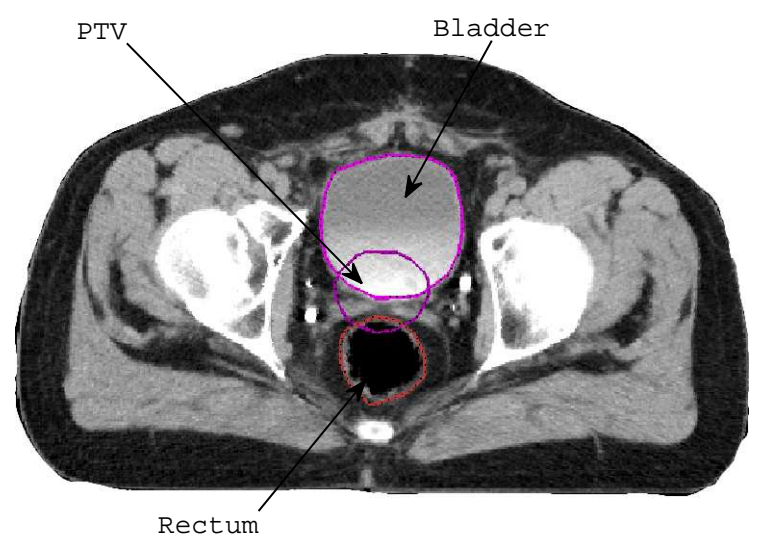

Figure 4: Transverse CT scan. 

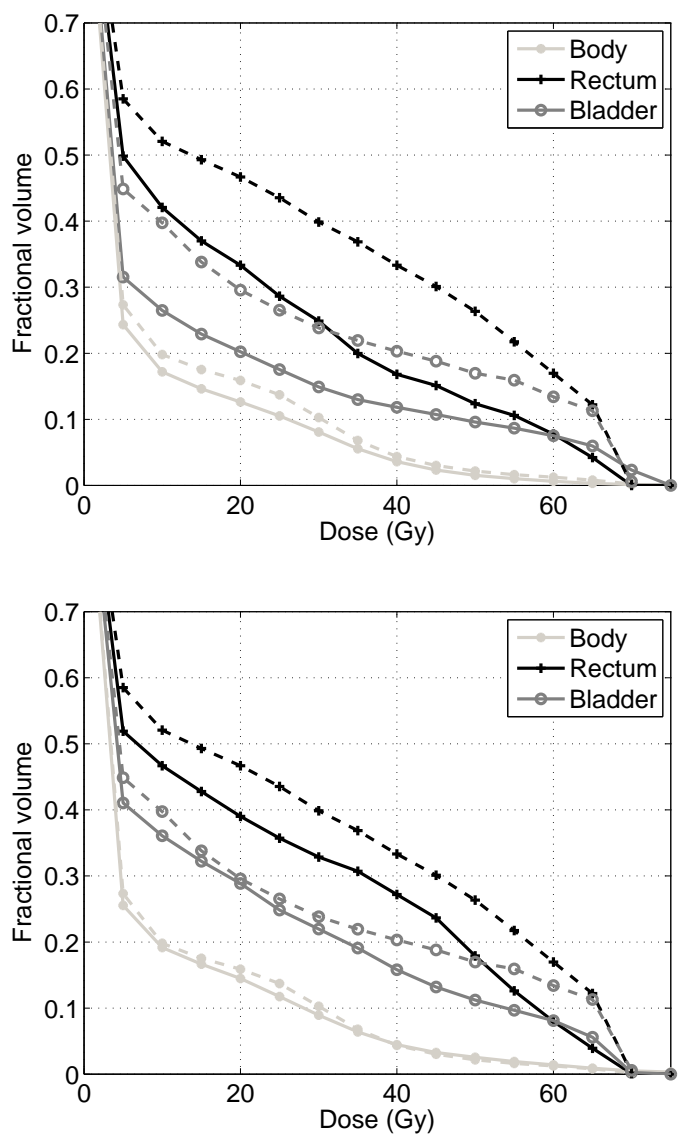

Figure 5: Dose-volume histograms for the organs at risk: bladder, rectum, and normal tissue. The dashed line corresponds to the conventional treatment (both figures), while the solid line corresponds to the expected value objective (left figure) and the CVaR objective (right figure). 


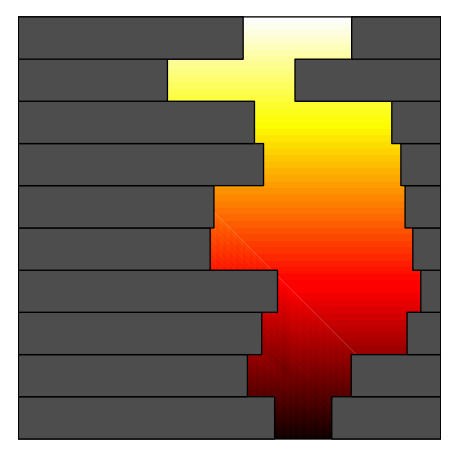

Multileaf collimators

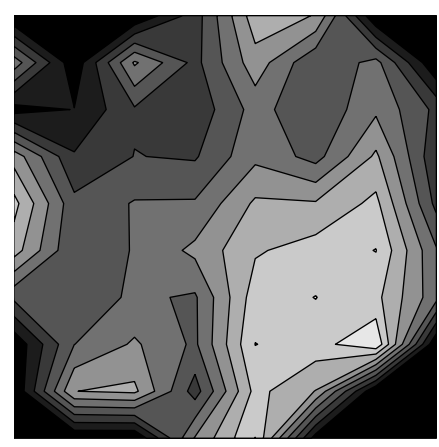

Beam fluence profile

Figure 6: Snapshot of the motion of the multileaf collimators (left figure) for the fluence (intensity) profile shown in the right figure for a cross-section of the beam. 


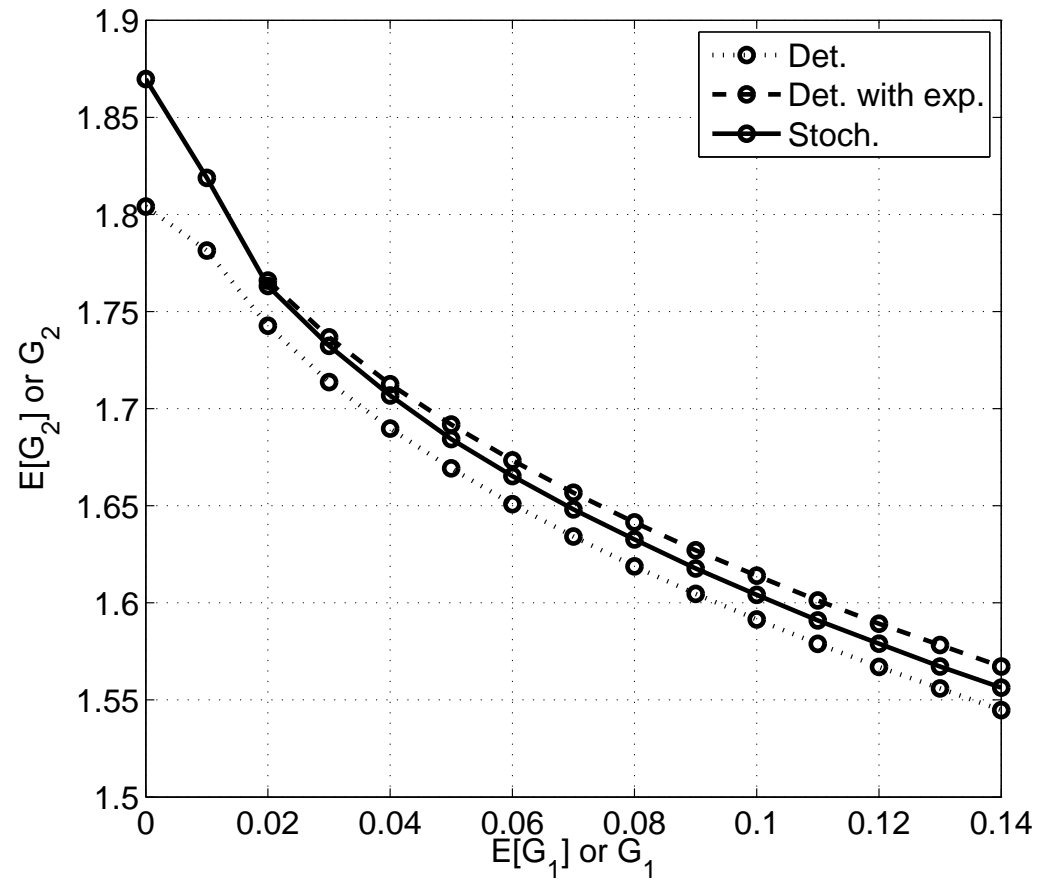

Figure 7: The figure shows three fronts: one is a local Pareto front for solutions $x_{s}^{*}$ to the stochastic model SDEL $\Omega$ (Stoch.); one is a local Pareto front for solutions $x_{d}^{*}$ to the corresponding deterministic model, i.e., with $c \equiv 0$ (Det.); and one is the deterministic solutions $x_{d}^{*}$ evaluated in $\mathrm{SDEL}_{\Omega}$ (Det. with exp.). 